\title{
Propagation Losses in Metal-Film-Substrate Optical Waveguides
}

\author{
ELSA M. GARMIRE AND H. STOLL
}

\begin{abstract}
The propagation losses in metal-film-substrate twodimensional optical waveguides are calculated. Losses for confined modes may become large and are at least an order of magnitude larger for TM than for TE modes. Higher order modes suffer more loss than the fundamental mode. Such mode-dependent loss can make efficient mode analyzers, useful for integrated optics modulation schemes.
\end{abstract}

\section{INTRODUCTION}

$\mathrm{P}$ LANAR films deposited on substrates have recently been shown to be very useful for waveguiding light, with promise for the fabrication of integrated optical circuits [1]-[5]. In this paper we shall explore optical propagation losses in planar film waveguides in the presence of a metal electrode, such as that required for electrooptic modulation. We consider a wide range of waveguide thicknesses and refractive indices and ignore scattering losses, which are negligible in semiconductor epitaxial layer waveguides [6]. For sufficiently thin films the optical propagation losses may be substantial; furthermore, the TM modes are at least ten times as lossy as TE modes. This suggests that a metal deposit on a waveguiding film can make an effective polarization analyzer, a convenient element to incorporate into integrated optical circuits.

Fig. 1 depicts the planar geometry. The spatial variation of the TE electric field for light of wavelength $\lambda$ in a guide $T$ wavelengths thick is given by

$$
x<0 \quad E_{y}(x, z)=A \exp \left(Q \frac{x}{\lambda T}-i B \frac{z}{\lambda T}\right)
$$

$0<x<\lambda T \quad E_{y}(x, z)$

$$
\begin{aligned}
= & {\left[C \exp \left(i H \frac{x}{\lambda T}\right)+D \exp \left(-i H \frac{x}{\lambda T}\right)\right] } \\
& \cdot \exp \left(-i B \frac{z}{\lambda T}\right)
\end{aligned}
$$

$x>\lambda T \quad E_{\iota}(x, z)=F \exp \left[-P\left(\frac{x}{\lambda T}-1\right)-\imath B \frac{z}{\lambda T}\right]$.

For TM modes replace $E_{y j}(x, z)$ with $H_{y}(x, z)$ and the form remains the same. The physical origin of the loss

Manuscript received August 9, 1971; revised January 31, 1972. This work was supported by the Office of Naval Research.

The authors are with the Department of Electrical Engineering, California Institute of Technology, Pasadena, Calif. we consider here is the absorption of the tail of the field profile that extends into the lossy metal. The metal is described by a complex refractive index $n_{1}=n-i k$, whose value is typically elose to those shown in Table I [7]. This introduces complex values into all the propagation equations. As a matter of notation we shall write all complex parameters in the form $H=H_{r}+i H_{i}$.

In this paper we shall make the assumption that the light intensity distribution goes to zero inside the metal in a distance much smaller than the waveguide thickness, i.e., $|H| \ll|Q|$. This condition is valid when the dielectric constant of the metal is sufficiently high. Numerically the requirement is ${ }^{1}$

$$
k \gg\left(\frac{m+1}{2}\right) \frac{1}{T}
$$

where $m$ is the waveguide mode order $(m=0,1,2, \cdots)$. This is easily fulfilled for the waveguides considered here, which are an appreciable fraction of a wavelength thick and contain only a few guided mode orders.

The numerical values for the dimensionless parameters $Q, H, P$, and $B$ are found by matching appropriate boundary conditions [8]. We calculate the propagation loss (i.e., $B_{i}$ ) from $H$, determined by the following transcendental equation:

$$
\begin{array}{r}
H=\tan ^{-1} \eta_{23} \sqrt{\frac{C_{23}{ }^{2}}{H^{2}}-1}+\tan ^{-1}\left(\frac{C_{21}}{H} \eta_{21}\right)+m \pi, \\
m=0,1,2, \cdots
\end{array}
$$

where the constants are $C_{21}=2 \pi T \sqrt{n_{2}^{2}-n_{1}^{2}} ; C_{23}=$ $2 \pi T \sqrt{n_{2}{ }^{2}-n_{3}{ }^{2}} \equiv 2 \pi T \sqrt{\Delta \epsilon} ; \eta_{21}=1, \eta_{23}=1$ for $\mathrm{TE}$ modes; and $\eta_{21}=n_{2}{ }^{2} / n_{1}{ }^{2}, \eta_{23}=n_{2}{ }^{2} / n_{3}{ }^{2}$ for TM modes. The complex propagation constant $B$ is determined from

$$
B^{2}=\left(2 \pi T n_{2}\right)^{2}-H^{2} .
$$

\section{Propagation Loss}

The aim of this paper is to determine the dependence of optical loss on waveguide thickness $T$ and dielectric discontinuity $\Delta \epsilon$. It is prohibitive to numerically calculate

1 This is derived from the relationship $\left.Q^{2}+H^{2}=C_{21^{2}}{ }^{1} \cdot Q^{2}\right\rangle$ $|H|^{2} \Rightarrow|H|^{2} \ll \mid C_{21}{ }^{2}$. From Table I observe that typically $k^{2} \gg n^{2}$ and $\left|n_{1}\right|^{2} \gg n_{2}{ }^{2}$. Thus $\left|C_{21}\right| \sim 2 \pi T^{2}$. Since $|H| \sim(m+1) \pi$, the condition is $[(m+1) / 2] \ll k T$. 


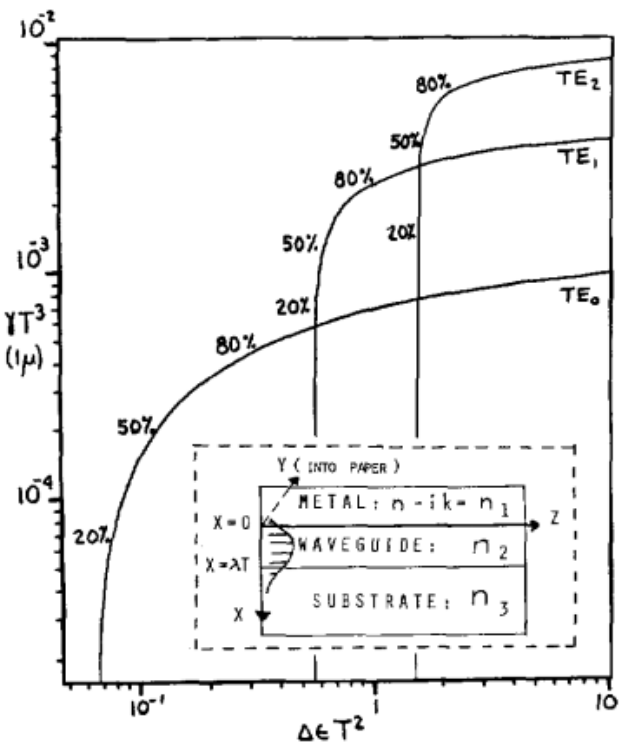

Fig. 1. Universal curves of waveguide loss as a function of dielectric constant for constant guide thickness; percentage of energy confined inside the guide region is indicated at typical points. Numbers on ordinate give loss for $1-\mu$ light guided in GaAs. Inset depicts geometry.

complex solutions to (3) for many values of $\Delta \epsilon$ and $T$. For TE modes we can obtain simple algebraic solutions and show that $B_{i} T^{2}$ is a universal function of $\Delta \epsilon T^{2}$ with a proportionality factor that depends only on refractive indices. This analysis is valid also for TM modes in relatively thick waveguides with moderate dielectric discontinuities.

The mathematical assumptions we shall use are

$$
\left|C_{21} \eta_{21}\right| \gg H_{r} \quad \eta_{23}=1 .
$$

For TE modes this is just the condition expressed in (2). ${ }^{1}$ For TM modes, the conditions can be written

$$
n_{2}-n_{3} \ll n_{2} \quad T \gg\left(\frac{m+1}{2}\right) \frac{k}{n_{2}^{2}} .
$$

That is, the diclectric discontinuity must be small and the guide must be thicker than some characteristic value. From the numbers in Table I we see that the latter condition is by no means always satisfied.

Using the assumption of (5), we may simplify (3) and (4), separate real and imaginary parts, and obtain

$$
\begin{aligned}
C_{23} & =\frac{H_{r}}{\sin H_{r}}(-1)^{m} \\
H_{i} & =\frac{H_{r} \operatorname{Im}\left(\frac{1}{C_{21} \eta_{21}}\right) C_{23} \cos H_{r}}{(-1)^{m}-C_{23} \cos H_{r}} \\
B_{i} & =-\frac{H_{i} H_{r}}{2 \pi T n_{2}} .
\end{aligned}
$$

We are intercsted in exploring the optical loss for a

\begin{tabular}{|c|c|c|c|c|c|c|c|c|}
\hline \multirow{2}{*}{$\begin{array}{c}\lambda \\
\text { microns }\end{array}$} & \multirow[b]{2}{*}{$n$} & \multirow[b]{2}{*}{$k$} & \multirow[b]{2}{*}{$n_{2}$} & \multicolumn{2}{|l|}{$10^{2} \kappa$} & \multirow[b]{2}{*}{$\delta_{1}$} & \multirow[b]{2}{*}{$\delta_{2}$} & \multirow[b]{2}{*}{$\delta_{3}$} \\
\hline & & & & TE & TM & & & \\
\hline 0.5 & $0 . \check{5}$ & 6 & 1.5 & 0.93 & $1 \overline{0}$ & 0 & 0.3 & 0.5 \\
\hline 1 & 1.8 & 10 & 3.3 & 0.55 & 5 & 0 & 0 & \\
\hline 10 & 25 & 67 & 3.3 & 0.16 & 70 & 1 & 1.5 & 1.75 \\
\hline
\end{tabular}
range of dielectric discontinuitics and waveguide thicknesses. Since we know $\left(m+\frac{1}{2}\right) \pi \lesssim H_{r} \lesssim(m+1) \pi$
TABLE I

Waveguide Parameters

for the $m$ th order mode, we specify a value for $H_{r}$, calculate appropriate waveguide thickness and dielectric discontinuity, and determine corresponding optical loss. This eliminates the need to solve a transcendental equation.

Writing the optical loss (units of inverse wavelengths) in terms of $H_{r}$,

$$
\gamma=\frac{B_{i}}{T}=\frac{\kappa}{T^{3}} \frac{H_{r}^{3} /(2 \pi)^{2}}{H_{\mathrm{r}}-\tan H_{r}}
$$

where

$$
\kappa_{\boldsymbol{Z}}=-\operatorname{Im}\left(\frac{1}{n_{2} \eta_{21} \sqrt{n_{2}{ }^{2}-n_{1}{ }^{2}}}\right) .
$$

Thus it is clear that $\gamma T^{3}$ is a universal function of $\Delta \epsilon T^{2}$ (i.e., $H_{r}$ ) with a shape that is independent of wavelength or refractive indices and a magnitude that is proportional to $\kappa$.

In Fig. 1 we show these loss curves $\gamma T^{3}$ as a function of $\Delta \epsilon T^{2}$ for the three lowest order modes. As $\Delta \epsilon T^{2}$ becomes small, the loss gocs to zero; this is waveguide cutoff $\left(H_{r} \rightarrow\left(m+\frac{1}{2}\right) \pi\right)$. Physically this reflects the fact that when the mode is not confined, the fraction of its power propagating in the lossy metal is negligible. This limit is not useful, however, since the fraction of energy propagating inside the guide becomes small. For convenience we have indicated this fraction at typical points in the figure.

For large values of $\Delta \epsilon T^{2}$ there is strong confinement of several modes. In this region $H_{r} \rightarrow(m+1) \pi$ and $\gamma T^{3}$ tend toward a constant value:

$$
\gamma T^{3} \rightarrow \kappa\left(\frac{m+1}{2}\right)^{2} .
$$

This formula allows us to make a quick estimate of the operating losses from the magnitude of $\kappa$. An approximate form of $\kappa$, valid when $\left|n_{1}\right|^{2} \gg n_{2}{ }^{2}$ and $k^{2} \gg n^{2}$, is given by

$$
\kappa=\left\{\begin{array}{ll}
\frac{n}{n_{2} k^{2}} & \mathrm{TE} \\
\frac{n}{n_{2}^{3}} & \mathrm{TM}
\end{array} .\right.
$$

Values for typical physical cases are shown in Table I. Recalling that $\gamma$ is expressed in units of inverse wavelengths, the optical loss is by no means small when the 
guide thickness becomes comparable to a wavelength. The numerical values of $\gamma T^{3}$ in Fig. 1 are given for TE light of wavelength $1 \mu$. From this graph it can be seen that a metal layer on a guide $1 \mu$ thick will introduce $\sim e^{-5}$ attenuation in $1 \mathrm{~cm}$ for TE modes of wavelength $1 \mu$.

From the loss estimate of (11), we observe that higher order modes suffer substantially more loss. Furthermore we observe that TM modes are much more lossy than TE modes [9]. For guides in which this simplified analysis describes TM modes, the ratio of TM to TE loss is $\kappa_{\mathrm{TM}} / \kappa_{\mathrm{TF}} \approx k^{2} / n_{2}{ }^{2}$. Observe, finally, that the losses are a very strong function of waveguide thickness and nearly independent of dielectric discontinuity (above cutoff).

We seek now to calculate the loss in waveguides that violate the conditions of $(6)$. In cases of interest, $H_{i} \ll$ $H_{r}$, and we write

$$
\begin{gathered}
H_{r}=\tan ^{-1} \eta_{23} \sqrt{\frac{C_{23}{ }^{2}}{I_{r}{ }^{2}}-1}+\operatorname{Re}\left(\tan ^{-1}\left(C_{21} \eta_{21} / H_{r}\right)\right)+m \pi, \\
m=0,1,2, \cdots \\
H_{i}=\frac{\operatorname{Im}\left(\tan ^{-1}\left(C_{21} \eta_{21} / H_{r}\right)\right)}{1+f^{-1}\left(C_{23}{ }^{2}-H_{r}{ }^{2}\right)^{-1 / 2}}
\end{gathered}
$$

where $f=\eta_{23}+\left(1 / \eta_{23}-\eta_{23}\right) H_{r}{ }^{2} / C_{23}{ }^{2}$. These equations hold only as long as $\mathrm{C}_{23}{ }^{2}-H_{r}{ }^{2} \gg H_{i}{ }^{2}$. Physically this means that the waveguide is above optical cutoff.

We solved the real transcendental (13) for $H_{r}$ by computer and then calculated $H_{i}$ and finally the optical loss $B_{i}=-H_{r} H_{i} / B_{r}$. The shape of these curves differs somewhat from the universal curves in Fig. 1. Nevertheless, 30-percent accuracy can be obtained with approximate scaling laws. We may use the universal curve for TM modes if the abscissa remains $\Delta \epsilon T^{2}$, but the ordinate becomes $\gamma T^{3-\delta}$. The value of $\delta$ was calculated to give approximate agreement for $T \lesssim 10$ and $\Delta \epsilon \lesssim 10$. Heuristic values of $\delta$ for the first three mode orders are shown in Table I. When $\delta=0,(10)$ can be used.

We shall show how to calculate numerically the TM loss in any waveguide of given $\Delta \epsilon$ and $T$ using the approximate scaling law. It is necessary first to scale the loss parameter $\gamma$ to a value of $T$ where the curves of the figure are valid. The region $T=10$ is valid for all cases of physical interest and we used this in estimating $\delta$. At $T=10$ we correlate TM loss for light at the wavelength of interest to TE loss for light of $1-\mu$ wavelength by the ratios of $\kappa$. Thus we have, for a guide of thicknoss $T$ and dielectric discontinuity $\Delta \epsilon$ :

$$
\left.\gamma T^{3}\right|_{\mathrm{TM}^{\lambda}}{ }^{\lambda}=\frac{\left.\kappa\right|_{\mathrm{TM}}{ }^{\lambda}}{\left.\kappa\right|_{\mathrm{TE}^{1 \mu}}}\left(\frac{T}{10}\right)^{\delta}\left(\left.\gamma T^{3}\right|_{\mathrm{TE}^{1 \mu}}\right)
$$

where $\left.\gamma T^{3}\right|_{T_{E}{ }^{1 \mu}}$ is read from the graph in Fig. 1 for the same value of $\Delta \epsilon T^{2}$. Note that for $10-\mu$ light, failure to incorporate $\delta$ into the calculation may introduce an error of almost two orders of magnitude.

The metal layer introduces a shift in the TM waveguide cutoff thickness of at most ${ }_{\mathrm{TM}} T_{c}-{ }_{\mathrm{T}} T_{c} \leq 1 / 4 \sqrt{\Delta \epsilon}$, an effect that was included in our computer calculations but will not be discussed in detail here.

\section{Mode Analyzer}

It has been shown that optical losses are higher for some modes than for others. This suggests that a metal film deposited on a planar waveguide may make an effective mode analyzer. Such an analyzer will be useful in integrated optics circuits as an important element of amplitude modulators. When a modulation signal is used to convert the waveguided light from one mode into another, this mode analyzer will selectively transmit only the original waveguide mode, resulting in amplitude modulation.

For example, the high ratio of TM to TE loss suggests a polarization analyzer. A metal-deposited guide 2.5 wavelengths thick with dielectric discontinuity $\Delta \epsilon=$ 0.048 and length $0.5 \mathrm{~cm}$ transmits ten times as much TE light at $1 \mu$ as TM light. Suppose the guide is composed of GaAs oriented along the [110] face; then a voltage applied across the guide produces the optimum conversion of one polarization into the other [10]. If light is coupled into such a waveguide modulator as the relatively lossless TE mode, an applied voltage will convert some of it into the lossy TM mode. Thus we have devised a variable loss, or amplitude modulator. An exact theory of such a modulator would require considering the effect of crystal anisotropy on the electromagnetic modes of the waveguide [11] and is beyond the scope of this paper. A rough estimate for bulk GaAs indicates that full conversion of one polarization into the other can be achieved in $0.5 \mathrm{~cm}$ with $5 \mathrm{~V}$ across the 2.5 - $\mu$-thick waveguide.

Another example of an intensity modulator utilizing mode-dependent losses involves variable mode conversion into higher order modes. It has been noted that the losses are proportional to $(m+1)^{2}$; in particular, the $m=1$ mode has four times the loss of the $m=0$ mode, if both are well confined. An effective intensity modulator would employ surface acoustic waves to couple $\mathrm{TM}_{0}$ modes into lossy $\mathrm{TM}_{1}$ modes in the presence of a metallic electrode in an arrangement similar to that used in [12]. An electrooptic phase grating [13] could also be employed to couple light into higher order modes.

The use of mode-dependent optical loss for intensity modulation will be most useful in the design of totally integrated optical circuits in which a modulator and detector are on the same chip. Then the grating coupler analyzers or external polarizers that have been used to date will not be employable.

\section{REFERENCES}

[1] R. Shubert and J. H. Harris, "Optical surface waves on thin films and their application to integrated data processors," IEEE Trans. Microwave Theory Tech., vol. MTT-16, pp. 1048-1054, Dec. 1968.

[2] S. Miller, "Integrated optics: An introduction," Bell Syst. Tech. J., vol. 48, p. 2059, 1969.

[3] D. Hall, A. Yariv, and E. Garmire, "Observation of propagation cutoff and its control in thin optical waveguides," Appl. Phys. Lett., vol. 17, p. 127, 1970. 
[4] J. E. Goell and R. D. Standley, "Integrated optical circuits," Proc. IEEE, vol. 58, pp. 1504-1512, Oct. 1970.

[5] P. K. Tien, "Light waves in thin films and integrated optics," Appl. Opt., vol. 10, p. 2395, 1971.

[6] D. Hall, A. Yariv, and E. Garmire, "Optical guiding and electro-optic modulation in GaAs epitaxial layers," Opt. Commun., vol. 1, p. 403, 1970.

[7] See, for example, Applied Optics and Optical Engineering, R. Kingslake, Ed. New York: Academic Press, 1965, p. 316.

[8] See, for example, W. W. Anderson, "Mode confinement and gain in junction lasers," IEEE J. Quantum Electron. vol. QE-1, pp. 228-236, Sept. 1965.

[9] Compare this result to that of J. Kane and H. Osterberg,
J. Opt. Soc. Amer., vol. 54, p. 347, 1964 (who found the TM mode was less lossy when $n_{2}$ was complex, but $n_{1}$ and $n_{3}$ were real).

[10] S. Namba, "Electro-optical effect of zincblende," J. Opt. Soc. Amer., vol. 51, p. 76, 1961.

[11] D. F. Nelson and J. McKenna, "Electromagnetic modes of anisotropic dielectric waveguides in $p-n$ junctions," J. Appl. Phys., vol. 38, p. 4057, 1967.

[12] L. Kuhn, P. F. Neidrich, E. G. Lear, and E. G. Lean, "Optical guided wave mode conversion by an acoustic surface wave," Appl. Phys. Lett., vol. 19, p. 428, 1971.

[13] J. M. Hammer, "Digital electro-optic grating deflector and modulator," Appl. Phys. Lett., vol. 18, p. 147, 1971.

\title{
Fluctuation Mechanism of Ultrashort Pulse Generation by Laser With Saturable Absorber
}

\author{
P. G. KRYUKOV AND VLADILEN STEPANOVICH LETOKHOV
}

\begin{abstract}
This paper presents a theoretical treatment of the fluctuation mechanism involved in the generation of picosecond laser pulses with saturable absorbers. The processes responsible for the shortening of the pulsewidth and for selection of the most intense pulse are treated. Some experimental results that confirmed the treatment are presented. The influence of inertia of saturable absorber and nonlinear losses (self-focusing and self-modulation) is discussed.
\end{abstract}

\section{INTRODUCTION}

$\mathrm{R}$ EMARKABLE progress in obtaining high-power short light pulses was achieved in 1966 [1], [2]. Measurements of pulse duration [3], [4] showed the ability of lasers with saturable absorbers to generate trains of pulses a few picoseconds in time duration. The study of emitted radiation from lasers having saturable absorbers in their feedback cavity was appreciably expanded after the introduction of the two-photon fluorescence method for time-duration measurements [5], [6]. The simplicity of this makes it very attractive. Unfortunately, the two-photon fluorescence method has no explicit definiteness, as it produces similar records for both mode-locked pulses and for irregular fluctuation spikes occuring from the multimode radiation from any laser $[7]-[9]$. The differenee lies in the value of the contrast ratio, which changes from 3 for an ideal ultrashort pulse train to 1.5 for incoherent multimode radiation (Gaussian noise) [8], [10], [11]. It is thus necessary

Manuscript received December 27, 1971.

P. G. Kryukov is with the P. N. Lebedev Physical Institute, Academy of Sciences, Moscow, USSR.

V. S. Letokhov is with the Institute of Spectroscopy, Academy of Sciences, Moscow, USSR. to measure the contrast ratio with very high accuracy if one has to be sure of the presence of ideal mode-locked pulses [12]. In addition, it was found that the actual pulse duration of the Nd:glass laser with a saturable absorber is an order of magnitude greater than the limiting duration defined by the spectral width of the radiation [2]. To explain this fact, the author of paper [13] has proposed a frequency "chirping" mechanism due to dispersion, which broadens the time duration of the subpicosecond light pulses. On the basis of this mechanism, the use of the pulse compression method to obtain shorter time-duration pulses [13], [14] was suggested. By contrast, in [15], [16] the authors succeeded in finding a subpicosecond structure within the ultrashort pulses with no compensation for the frequency chirping. Many papers, besides those mentioned here, have been concerned with the study of these two facts. Detailed information on the question may be found in [17], [18].

Far less attention has been paid to the study of the dynamics of ultrashort pulse formation process. Creation of the regular train of ultrashort pulses was considered to be an evident process, the so-called "self-mode locking by a saturable absorber." Prcviously, mode locking in gas and solid-state lasers by intracavity active-loss modulation was studied [19]-[21]. In this case, the periodic disturbance of the resonator gradually increases the number of modes and, finally, results in the strict periodic sequence of pulses with time duration $\tau_{p} \simeq T / m$, where $m$ is a number of locked modes and $T$ is the repetition period. A laser utilizing a saturable absorber also emits a strictly periodic sequence of very short pulses with a broad radiation spectrum. This formal resemblance gives 